\title{
Current understanding and management of pulmonary Langerhans cell histiocytosis
}

\author{
Robert Vassallo, ${ }^{1}$ Sergio Harari, ${ }^{2}$ Abdellatif Tazi ${ }^{3}$
}

${ }^{1}$ Departments of Medicine, Physiology and Biomedical Engineering, Mayo Clinic, Rochester, Minnesota, USA ${ }^{2}$ U.O. di Pneumologia e Terapia Semi-Intensiva Respiratoria "Servizio di Fisiopatologia Respiratoria ed Emodinamica Polmonare, Ospedale San Giuseppe" Multimedica IRCCS, Milano, Italy

${ }^{3}$ Department of Pulmonary Medicine, Saint-Louis Hospital, National Reference Center for Langerhans Cell Histiocytosis, University Paris Diderot Sorbonne Paris Cite, Inserm UMR-1153 (CRESS), Biostatistics and Clinical Epidemiology Research Team (ECSTRA), Paris, lle-de-France, France

\section{Correspondence to}

Dr Robert Vassallo, Division of Pulmonary and Critical Care Medicine, Departments of Medicine, Physiology and Biomedical Engineering, Mayo Clinic, Rochester, Minnesota 55905, USA; vassallo.robert@ mayo.edu

Received 10 February 2017 Revised 10 May 2017 Accepted 15 May 2017 Published Online First 8 July 2017

\begin{abstract}
Pulmonary Langerhans cell histiocytosis (PLCH) is a diffuse lung disease that usually affects young adult smokers. PLCH affects different lung compartments; bronchiolar, interstitial and pulmonary vascular dysfunction may coexist to varying extents, resulting in diverse phenotypes. Analyses of PLCH tissues have identified activating mutations of specific mitogenactivated protein kinases (BRAF ${ }^{\mathrm{V} 600 \mathrm{E}}$ and others). The current consensus is that PLCH represents a myeloid neoplasm with inflammatory properties: the myeloid tumour cells exhibit surface CD1a expression and up to $50 \%$ of the cells harbour activating BRAF or other MAPK mutations. PLCH may be associated with multisystem disease. The detection of disease outside of the thorax is facilitated by whole body positron emission tomography. The natural history of PLCH is unpredictable. In some patients, disease may remit or stabilise following smoking cessation. Others develop progressive lung disease, often associated with evidence of airflow limitation and pulmonary vascular dysfunction. Due to the inability to accurately predict the natural history, it is important that all patients undergo longitudinal follow-up at least twice a year for the first few years following diagnosis. The treatment of PLCH is challenging and should be individualised. While there is no general consensus regarding the role of immunosuppression or chemotherapy in management, selected patients may experience improvement in lung function with therapy. Determination of BRAF ${ }^{\mathrm{V} 600 \mathrm{E}}$ or other mutations may assist with the development of an individualised approach to therapy. Patients with progressive disease should be referred to specialised centres and considered for a trial of pharmacotherapy or evaluated for transplantation.
\end{abstract}

\section{INTRODUCTION}

Langerhans cell histiocytosis $(\mathrm{LCH})$ is a rare disease of unknown aetiology, characterised by organ infiltration with specialised myeloid cells that share morphological and surface receptor markers with epidermal Langerhans cells (LCs). ${ }^{12}$ LCH belongs to the broader group of histiocytic disorders, comprising a diverse collection of diseases, whose common denominator is the accumulation of cells of the reticuloendothelial system in the tissues and organs concerned. ${ }^{2}$

LCH can affect patients of all ages. The Histiocyte Society classifies clinical forms of $\mathrm{LCH}$ according to the number and type of organs involved. ${ }^{2}$ It distinguishes systemic $\mathrm{LCH}$, combining varying degrees of bone, skin, hypothalamic-pituitary, lymph node, lung lesions, and more rarely central neurological lesions. Systemic LCH is associated with a worse prognosis when so-called 'risk organs' are involved (liver, spleen, haematological involvement). This acute form of systemic LCH (previously called Letterer-Siwe syndrome) is predominantly observed in very young children. ${ }^{2}$ Localised forms of $\mathrm{LCH}$ (previously called eosinophilic granuloma) often affect bone, skin and the lungs, and frequently have a more indolent course with the potential for spontaneous remission. ${ }^{2}$ However, patients with an initially localised form of $\mathrm{LCH}$ can subsequently develop systemic LCH. Pulmonary involvement can be present in systemic forms of LCH, but more commonly pulmonary LCH (PLCH) occurs in young adult smokers and is usually isolated to the chest. ${ }^{3}$

\section{EPIDEMIOLOGY}

The prevalence of PLCH is unknown but may account for about 3-5\% of all adult diffuse lung diseases. The precise prevalence of PLCH may be higher than estimated in earlier studies, ${ }^{4}$ because it may be asymptomatic, may spontaneously remit, and may be difficult to identify in very advanced forms. While infrequent in individuals of African origin, PLCH has been well described in Asian subjects. ${ }^{5}$ In adults, PLCH occurs predominantly in young smokers or ex-smokers ( $>90 \%$ of cases), with a peak incidence between the ages of 20 and 40 years. $^{3}$ PLCH occurs with equal frequency in both genders. ${ }^{36}$ Other inhalation exposures may be relevant; for example, about $20 \%$ of patients of the French LCH registry are also cannabis users (personal observation).

\section{PATHOGENESIS}

There are four key elements concerning the pathogenesis of PLCH: (1) the mechanisms of accumulation of large numbers of CD1a+ cells in bronchiolocentric loosely formed granulomas; (2) the capacity of these granulomatous lesions to destroy and remodel surrounding tissues; (3) the reactive versus clonal/neoplastic nature of the disease; and (4) the role of smoking in adult PLCH.

\section{Accumulation of CD1a+ cells}

The accumulation of CD1a+ cells resulting in loosely formed granulomas around small airways likely results from recruitment of circulating peripheral blood myeloid haematopoietic precursor cells, which then differentiate in the tissues involved. Essential for this differentiation are growth factors (granulocyte macrophage colony stimulating factor: GM-CSF) and chemokines (CCL20 and CCL2) known to be expressed around PLCH lesions. ${ }^{7} 8$ Differentiation of precursor cells residing locally in
To cite: Vassallo R, Harari $S$,

2017:72:937-945 
the tissues involved is also possible. A proportion of granuloma cells express CD1a but not langerin (marker of LCs), indicating dendritic cells at various stages of differentiation. Local neoangiogenesis, signalling and cell adhesion molecules are involved in CD1a+-cell, T-cell and other inflammatory cell accumulation. ${ }^{9}$ The CD1a + cells in PLCH granulomas do not bear markers of proliferation. ${ }^{1011}$ In contrast, these cells appear to be less sensitive to apoptosis. ${ }^{12}$

\section{Destructive nature of PLCH granulomas}

A specific characteristic of PLCH granulomas is their capacity to destroy and remodel surrounding tissues. ${ }^{13}$ The lesional CD1a+ cells within granulomas have a different phenotype from the same cells under physiological conditions. Transcriptome studies of langerin-positive cells obtained from LCH granulomas show that these cells have a different transcriptomic profile from both epidermal LCs and normal dendritic cells. ${ }^{14}$ CD1a+ cells of PLCH granulomas express, to varying degrees, membrane maturation markers (especially co-stimulation molecules) similar to those present on the surface of dendritic cells after exposure to pathogens or activating cytokines. ${ }^{915}$

Although bearing an activated surface phenotype, CD1a+ cells extracted from LCH granulomas appear to be functionally impaired. ${ }^{16}$ Thus, although T lymphocytes are abundant in $\mathrm{LCH}$ granulomas, it is unlikely that the observed tissue destruction is secondary to a local cytotoxic immune reaction. Furthermore, a considerable proportion of the $\mathrm{T}$ lymphocytes that infiltrate LCH granulomas are regulatory $\mathrm{T}$ lymphocytes. ${ }^{10}$ In contrast, various metalloproteinases have been identified in LCH granulomas and could account for LCH-induced tissue destruction. ${ }^{17} \mathrm{~A}$ role for interleukin-17 in tissue remodelling has been suggested by Coury et al,$^{18}$ although other investigative teams have not confirmed those findings. ${ }^{19}$ More recently, activation of the Notch1 signalling pathway has been shown to be at least partly responsible for the specific profile of LCH cells. ${ }^{20}$

\section{PLCH: reactive, clonal or neoplastic?}

Whether PLCH is a reactive or clonal process has been a subject of debate since this disease was first identified. The prevailing thinking until recently was that PLCH represents an inflammatory reactive disorder induced by cigarette smoke. In support of this contention were the observations that mitotic figures and recurrent cytogenetic abnormalities had not been observed in the CD1a+ cells of these lesions. ${ }^{11}$ The 'spontaneous' resolution observed in certain PLCH cases - often following smoking cessation $^{2122}$ - as well as the detection of substantial infiltrating immune cells in PLCH granulomas, ${ }^{15}$ also were considered arguments favouring a non-clonal reactive immune granulomatous reaction. Conversely, the occasionally aggressive nature of the disease and the efficacy of chemotherapy in severe systemic forms of PLCH favoured a neoplastic mechanism. Discordant results have been reported concerning the clonal nature of $\mathrm{CD} 1 \mathrm{a}+$ cells in the different forms of LCH: a study of CD1a+ cells derived from extrapulmonary lesions of focal as well as systemic forms of LCH (the majority of the subjects were children) reported the clonal nature of these cells, ${ }^{23}$ while a study conducted on lung biopsies from 13 women with PLCH using an alternative (and potentially less sensitive) molecular approach to determine clonality showed that $70 \%$ of the lesional CD1a+ cells were polyclonal in that small population of adults with PLCH. ${ }^{24}$

The recent identification of recurrent genetic abnormalities involving cellular proliferative pathways in lesional CD1a+ cells obtained from systemic $\mathrm{LCH},{ }^{25}$ as well as pulmonary $\mathrm{LCH}^{26}$ tissues, provides the strongest argument to date in favour of the neoplastic hypothesis for LCH. ${ }^{27}{ }^{28}$ The most prevalent genetic abnormality described in $\mathrm{LCH}$ is the $\mathrm{BRAF}^{\mathrm{V} 600 \mathrm{E}}$ mutation, which also occurs in various types of cancers, including malignant melanomas and almost all cases of hairy cell leukaemia ${ }^{29}$ (and has also been reported in benign naevi). ${ }^{30}$ Functionally, this $\mathrm{BRAF}^{\mathrm{V} 600 \mathrm{E}}$ mutation results in a modified kinase domain that is associated with constitutive activation of the mitogen-activated protein kinase (MAPK) pathway which plays a key role in cell differentiation and survival (figure 1). This mutation occurs in $35-50 \%$ of people with $\mathrm{PLCH},{ }^{26}$ and at least $50 \%$ of those with systemic LCH granulomas. ${ }^{25}$ Interestingly, Berres et $a l^{31}$ showed that in children with severe systemic forms of $\mathrm{LCH}$, the presence of the $\mathrm{BRAF}^{\mathrm{V} 600 \mathrm{E}}$ mutation was not only present in tissue lesions (somatic mutation), but also in circulating and sometimes bone marrow precursors of dendritic cells that infiltrate $\mathrm{LCH}$ granulomas. Further evidence supporting a central role for this specific mutation in the pathogenesis of $\mathrm{LCH}$ comes from the recent report showing pathological lesions resembling systemic LCH in a murine model in which dendritic cells expressed the $\mathrm{BRAF}^{\mathrm{V} 600 \mathrm{E}}$ mutation. $^{31}$

While approximately half of PLCH cases are associated with wild-type BRAF status, activation of the MAPK pathway - as determined by immunohistochemical localisation of phosphorylated MAPK pathway members - occurs in all cases. ${ }^{25}$ This implies additional mechanisms for MAPK pathway activation in the absence of $\mathrm{BRAF}^{\mathrm{V} 600 \mathrm{E}}$ mutations. Indeed, MAP2K1 as well as activating NRAS ${ }^{\mathrm{Q} 61 \mathrm{~K} / \mathrm{R}}$ mutations have been reported. ${ }^{32-34}$ While in systemic LCH, BRAF and MAP2K1 mutations are mutually exclusive, ${ }^{32}$ NRAS mutations occurred concurrently with $\mathrm{BRAF}^{\mathrm{V} 600 \mathrm{E}}$ mutations in PLCH and both mutations were carried by different cell clones. ${ }^{34}$ Importantly, NRAS ${ }^{\mathrm{Q} 61}$ mutations were not identified in non-pulmonary LCH lesions, and in a singular case occurred in pulmonary but not in skin lesions from the same patient. $^{34}$

The clinical significance of MAPK pathway mutations in PLCH is not fully appreciated. When first reported, patients with $\mathrm{LCH}$ lesions harbouring the $\mathrm{BRAF}^{\mathrm{V} 600 \mathrm{E}}$ mutation were younger than patients without somatic BRAF mutations, but no link was observed with disease phenotype. ${ }^{25}$ In the study by Berres et al, ${ }^{31}$ the presence of somatic $\mathrm{BRAF}^{\mathrm{V} 600 \mathrm{E}}$ mutations was associated with increased risk of disease recurrence in paediatric systemic $\mathrm{LCH}$, but did not influence survival. In contrast to Roden et al, ${ }^{26}$ the study by Mourah $e t a l,{ }^{34}$ did not find a correlation between smoking and the presence of BRAF or NRAS mutations. In that study, univariate analysis demonstrated an association between the presence of NRAS mutations in lung lesions and the risk of spontaneous progression of PLCH. ${ }^{34}$ In children with LCH, the $\mathrm{BRAF}^{\mathrm{V} 600 \mathrm{E}}$ mutation is associated with high-risk disease and increased resistance to first-line therapy. ${ }^{28}$ Further studies are needed to evaluate the impact of MAPK mutations and the clinical phenotype of $\mathrm{LCH}$ and to determine if these mutations are useful for disease stratification and outcome, particularly in adults.

\section{Role of smoking}

Several lines of evidence support a role for cigarette smoking in the pathogenesis of adult PLCH. It is noteworthy that children with extrapulmonary LCH who subsequently develop PLCH during adolescence or adulthood are often smokers. ${ }^{35}$ Although the precise mechanisms by which tobacco smoke is involved in the pathogenesis of PLCH remains incompletely understood, smoking-induced changes in the epithelium of 


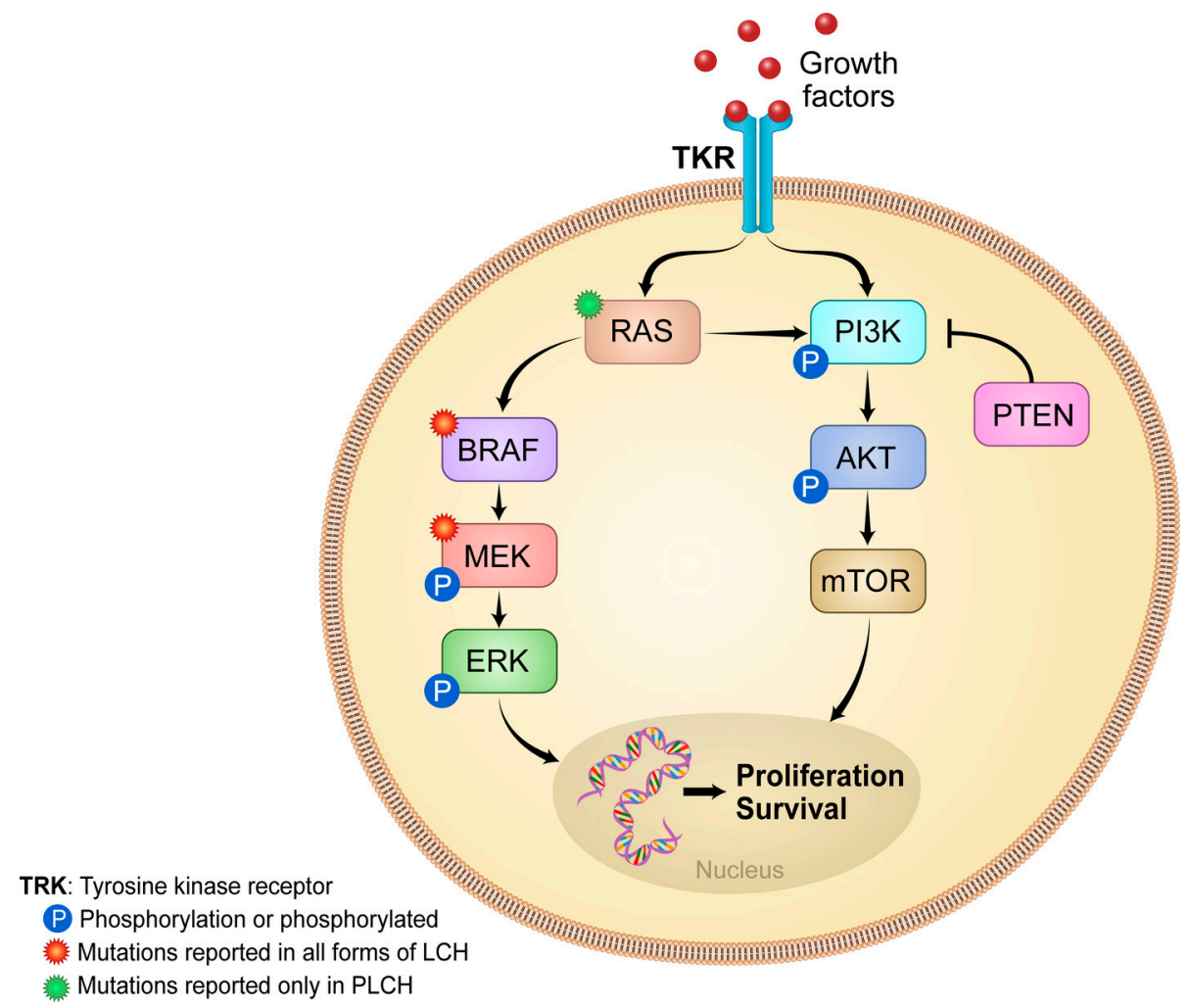

Figure 1 Cellular signals mediated by binding of growth factors to respective tyrosine kinase receptors (TKRs) activate downstream cellular signalling pathways such as the mitogen-activated protein kinase (MAPK) and other pathways. These pathways mediate a number of cellular responses, including cell proliferation and survival/death pathways. Constitutively active mutated BRAF and MEK variants have been reported to occur in both systemic Langerhans cell histiocytosis (LCH) and pulmonary LCH (PLCH), while active RAS mutations have only been described in PLCH.

distal bronchioles are likely involved. ${ }^{36} 37$ Smoking induces accumulation of CD1a+ cells in the lungs in healthy smokers, in various diseases and in murine models. ${ }^{38}$ Smoking stimulates local production of cytokines, which are important for the recruitment, differentiation and activation of dendritic cells, especially tumour necrosis factor $\alpha$ (TNF $\alpha)$, granulocyte macrophage colony-stimulating factor (GM-CSF), transforming growth factor $\beta$ (TGF $\beta$ ) and chemokine CCL20, which are also expressed in PLCH lesions. ${ }^{76-38}$ Tobacco smoke also promotes the survival of dendritic cells via anti-apoptotic mechanisms. ${ }^{12} 39$ Osteopontin appears to play a role in PLCH, as large quantities of osteopontin are present in the bronchoalveolar lavage (BAL) of patients with PLCH compared with control smokers. ${ }^{40}$ This glycoprotein, the secretion of which is increased by nicotine, exerts chemoattractant effects on monocytes/macrophages and dendritic cells. ${ }^{40}$ In a rat model, osteopontin overexpression induces lesions similar to those of PLCH. ${ }^{40}$ Whether cigarette smoke can directly induce mutations in the MAPK pathway has not been demonstrated, but it is possible that smoking is the necessary stimulus that enables the recruitment and persistence of myeloid cell clones harbouring MAPK mutations in the lung of smokers with PLCH. What drives the inflammatory and granulomatous like nature of the nodular lesions that characterise PLCH remains unknown: potentially cigarette smoke injury to distal lung epithelial structures together with the induction of myeloid cell clones harbouring mutated MAPKs may be the critical events that set the stage for the development of the nodular early lesions. Chilosi et $a l^{41}$ recently proposed oncogene-induced senescence as a mechanism by which cell cycle arrest and inflammation may be perpetuated in PLCH. Utilising an immunohistochemical approach to detected $\mathrm{BRAF}^{\mathrm{V} 600 \mathrm{E}}$, the authors showed immunoreactivity in 12 of 19 PLCH biopsies. ${ }^{41}$ Interestingly, a high proportion of the CD1a+ cells in those cases showed expression of $\mathrm{p} 16^{\mathrm{INK} 4 \mathrm{a}}$ and $\mathrm{p} 21^{\mathrm{CIP} 1 / \mathrm{WAF} 1}$ (markers of senescence), suggesting that PLCH may be a senescence-related neoplasm. ${ }^{41}$ In PLCH, it is possible that either the $\mathrm{BRAF}^{\mathrm{V} 600 \mathrm{E}}$ - or an alternative MAPK mutation - can lead to activation of senescence pathways in CD1a+ myeloid cells that go on to infiltrate the lung. It is also possible that cellular senescence - potentially driven by cigarette smoke exposure - may induce the senescence-associated secretory phenotype which is recognised as a chronic proinflammatory stimulus. This in turn may act as the 'driver' of the inflammatory cell recruitment and inflammation noted in the PLCH nodular lesions. While cigarette smoke is clearly associated with PLCH, it is noteworthy that in Ercheim-Chester Disease (ECD) - the other histiocytic disorder in which somatic BRAF and MAPK mutations are very prevalent ${ }^{2}-$ is more frequently observed in non-smokers. ${ }^{42}$

The rarity of PLCH relative to the prevalence of smoking in the general population suggests the presence of predisposing factors in patients who develop this disease. Another exogenous factor, for example viral infection, is another potential cofactor involved in the pathogenesis of PLCH in genetically predisposed patients. Various studies designed to detect the pathogens responsible have generally been inconclusive.

\section{CLINICAL PRESENTATION}

Adult PLCH is generally diagnosed in three main settings ${ }^{35}{ }^{43}$ :

1. Onset of respiratory symptoms (usually cough and dyspnoea) in about two-thirds of patients. Constitutional symptoms 


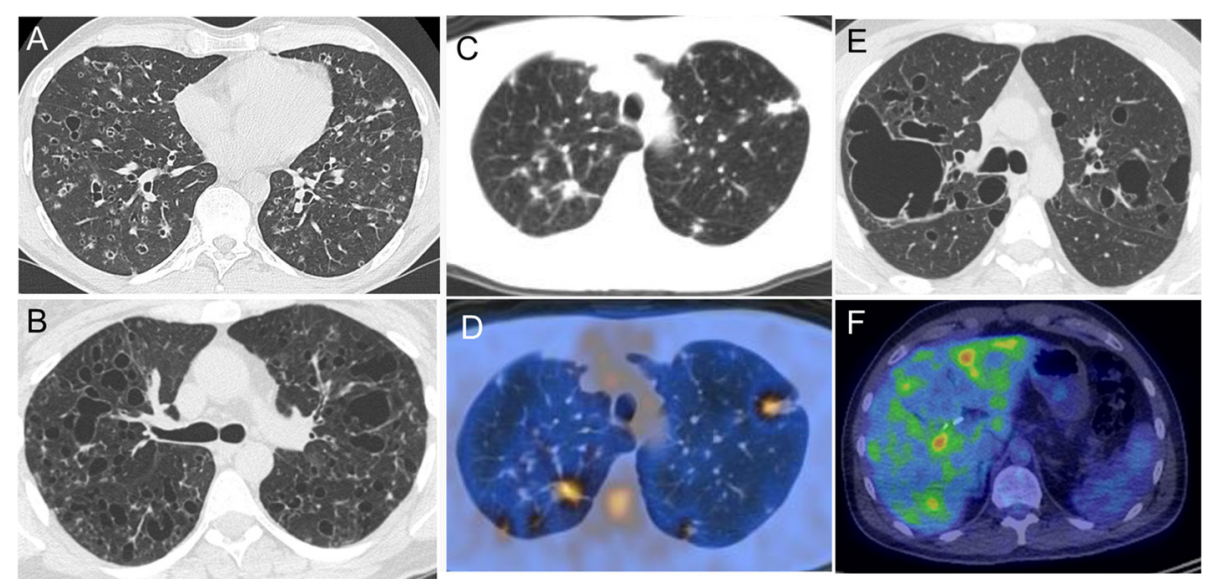

Figure 2 (A) Representative high-resolution chest CT image from a patient with biopsy-proven pulmonary Langerhans cell histiocytosis (PLCH) demonstrating a combination of small nodules, cavitary nodules and lung cysts. (B) Representative chest CT image from a patient with advanced biopsy-proven PLCH demonstrating cystic lung lesions of varying sizes and appearance. Nodular change is not apparent. (C and D) Corresponding chest CT and positron emission tomography (PET) images from a patient with biopsy-proven PLCH showing nodular lesions on the chest CT which are hypermetabolic on the PET. (E) Representative chest CT image from a patient with biopsy-proven systemic Langerhans cell histiocytosis with pulmonary, liver and bone involvement. The chest CT image demonstrates cystic changes with formation of a bullous like abnormality in the right upper lobe. (F) Representative PET image showing hypermetabolic liver lesions from the same patient whose chest CT is shown in panel E.

(fever, malaise, sweats and weight loss) are described in 15$20 \%$ of cases.

2. Acute presentation with a spontaneous pneumothorax in about $15-20 \%$ of cases. Spontaneous pneumothorax can occur at any time during the course of disease; it may be bilateral and recurrent.

3. As an incidental finding on routine chest X-ray in $5-25 \%$ of cases.

Haemoptysis occurs rarely and justifies the search for possible complications (infectious bronchitis, lung cancer, rarely aspergillus colonisation of a cystic cavity) or an alternative diagnosis. Adult PLCH is generally isolated. When present, extrathoracic lesions usually involve bone, the hypothalamic-pituitary axis (polyuria-polydipsia syndrome due to diabetes insipidus) and more rarely the skin. ${ }^{3}$ Physical examination is generally normal, except in advanced stages or when associated with extrathoracic involvement.

\section{CHEST IMAGING}

Chest X-ray often shows bilateral, and generally symmetric, reticulo-micronodular changes, in which cysts may sometimes be identified, predominantly involving the upper and middle lung fields. ${ }^{44}$ Occasionally, chest radiography may reveal a pneumothorax, or rarely, a lytic lesion of a rib. Pleural effusion and mediastinal lymphadenopathy are very uncommon. In rare cases, the chest X-ray is normal. ${ }^{44}$

Chest high-resolution computed tomography (HRCT) characteristically demonstrates a combination of nodules, cavitating nodules measuring $1-10 \mathrm{~mm}$ in diameter and thick-walled or thin-walled cysts (figure 2). ${ }^{45-48}$ Cysts vary in size and may coalesce to form irregular shapes (figure 2). Abnormalities are predominantly located in the upper and middle lung fields with relative sparing of the lung bases. The types of lung lesions vary with disease duration. In early disease, nodules and cavitated nodules are more numerous than lung cysts, while more advanced disease is often cystic in appearance. ${ }^{47}$ Significantly enlarged mediastinal lymph nodes are rarely observed. In some cases there are extensive ground glass opacities due to the concomitant presence of other smoking-related interstitial lung disease (ILD). ${ }^{49}$
Quantitative assessment of cystic lung disease on HRCT also correlates with pulmonary function parameters. ${ }^{50}$

The role of positron emission tomography (PET)-CT in the assessment of isolated PLCH is not well defined. Pulmonary nodules can be hypermetabolic, in which case they cannot be distinguished from malignant disease (figure 2). ${ }^{51}$ Thickwalled cysts can sometimes also demonstrate high metabolic uptake. ${ }^{51}$ The presence of an isolated hypermetabolic pulmonary nodule in a patient who smokes may be due to PLCH, but should raise suspicion for a surperimposed lung cancer which needs to be carefully excluded depending on the clinical context. Whether PET-CT is predictive of the natural disease course or response of PLCH to treatment remains to be determined. ${ }^{52}$

\section{PULMONARY FUNCTION TESTS}

Pulmonary function abnormalities vary according to the extent of cystic involvement and disease duration. The most common abnormality is reduction of diffusing capacity of the lungs for carbon monoxide (DLCO) (observed in $80-90 \%$ of cases). ${ }^{3}$ In addition, the pulmonary function test often demonstrates reduction in vital capacity (VC), normal or increased residual volume (RV), preserved total lung capacity (TLC) and increased or normal RV/TLC ratio (air trapping). ${ }^{53}$ An obstructive ventilatory defect is observed in approximately a third to half of all patients, while restrictive ventilatory defect (defined by a TLC $<80 \%$ predicted) is present in a minority (usually <20\%). ${ }^{354}$ Mixed restrictive and obstructive abnormalities may also be seen in about a third of patients. A proportion of patients will have normal lung volumes and spirometry with an isolated reduction in diffusing capacity to carbon monoxide. In a recent study, $10 \%$ of patients with obstructive physiology demonstrated reversibility following short-acting $\beta 2$-agonist therapy. ${ }^{54}$ The severity of airflow limitation on lung function testing correlates with the extent of cystic lesions on HRCT. ${ }^{55}$

\section{BRONCHOSCOPY AND BRONCHOALVEOLAR LAVAGE}

Bronchoscopy is macroscopically normal or reveals non-specific smoking-related abnormalities. Bronchial mucosal biopsies 


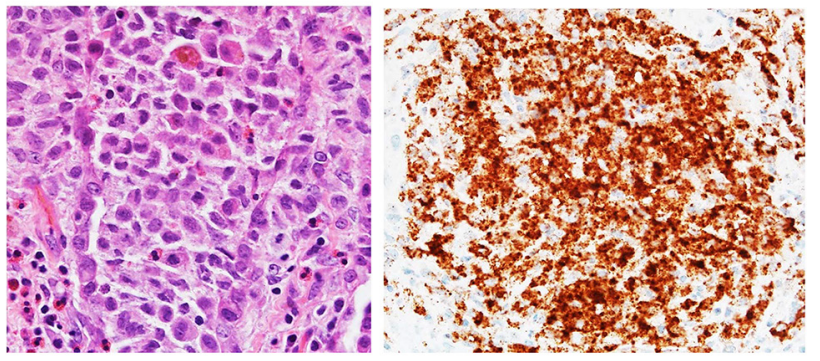

Figure 3 Light microscopic features of cellular pulmonary Langerhans cell histiocytosis is shown in the left-hand panel. The cellular infiltrate includes cells that morphologically resemble Langerhans cells and are characterised by delicate and folded nuclei. Scattered eosinophils are present (hematoxylin and eosin, $\times 400$ ). The right-hand panel demonstrates cellular staining with a BRAF-V600E antibody on a separate biopsy specimen, showing marked positivity for the BRAFV600E antigen in cells (magnification $\times 400$ ).

do not contribute to the diagnosis of PLCH, but may be useful to exclude other diseases. Transbronchial lung biopsies have variable diagnostic yields (15-40\%) due to the focal nature of lesions. ${ }^{5657}$ Cryobiopsy is associated with an increased diagnostic yield due to the larger samples acquired. ${ }^{58}$ BAL may be useful to support the diagnosis of PLCH by showing an increase in the number of CD1a+ cells. ${ }^{59}$ The presence of at least $5 \%$ of CD1a+ cells in BAL has only been reported in PLCH, but is observed in only a minority of cases. ${ }^{5760} \mathrm{BAL}$ is therefore rarely diagnostic of PLCH in adults.

\section{LUNG PATHOLOGY}

The histological hallmark of PLCH is accumulation of CD1a+ cells organised into loosely formed granulomas, preferentially located in, and destroying the wall of distal bronchioles. ${ }^{61} \mathrm{On}$ light microscopy, LCs may be identified by their convoluted nucleus, pale, slightly eosinophilic cytoplasm, and an irregular indented nucleus (figure 3). On immunohistochemical examination, they express CD1a and langerin (CD207). Langerin has replaced the search for Birbeck granules on electron microscopy, which is now rarely performed. In contrast, cytoplasmic expression of protein S100 is non-specific. Immunohistochemical detection of $\mathrm{BRAF}^{\mathrm{V} 600 \mathrm{E}}$ expression is a simple and effective method to screen for this mutation when the diagnosis is confirmed by tissue biopsy (figure 3 ).

PLCH granulomas are focal, poorly demarcated, separated by zones of healthy tissue, and are centred on distal bronchioles (terminal and respiratory), accompanying arterioles and sometimes adjacent venules. ${ }^{61}$ The appearance of PLCH granulomas depends on the stage of the disease, and lesions of different ages are often present on the same lung biopsy. New lesions are localised in the distal bronchiolar wall, which appears to be initially infiltrated by variable numbers of LCs and lymphocytes, monocytes/macrophages and subsequently inflammatory cells, especially eosinophils and more rarely giant cells. ${ }^{61}$

Destruction of bronchiolar epithelium occurs early during the disease process, making it difficult to confirm the bronchiolocentric distribution of the lesions (presence of a residual ring of smooth muscle cells) on a single tissue section. Examination of serial sections with three-dimensional reconstructions demonstrated that PLCH lesions progress in the form of a granulomatous cuff along the bronchiole. ${ }^{62}$ Cystic lesions are due to destruction of the bronchiolar wall and progressive dilatation of the bronchiolar lumen.
With progression of the lesions, the number of CD1a+ cells decreases, while inflammatory cells persist, associated with lymphoid aggregates, which are subsequently replaced by either fibrosis in the form of a characteristic stellate scar, or contiguous and confluent cystic cavities, surrounded by a fibrous ring. ${ }^{61}$ Interestingly, correlations between CT features and pulmonary histopathology have shown that LCH granulomas may still be observed in diffuse cystic forms and that inflammatory cells may persist even inside thin-walled cysts. ${ }^{63}$

In areas not involved by the disease process, lung architecture is preserved, but non-specific smoking-related lesions (respiratory bronchiolitis, intra-alveolar accumulation of pigmented macrophages, lymphoid cells) are often present. In some cases, the predominant features may be suggestive of respiratory bronchiolitis ILD or desquamative interstital pnemonia, but underlying specific LCH granulomas should be sought by comprehensive examination of biopsy samples, including immunohistochemistry. ${ }^{49}$

The differential diagnosis includes other histiocytic and eosinophilic lung diseases, as well as desquamative interstitial pneumonia, hypersensitivity pneumonitis and idiopathic interstitial pneumonias. ${ }^{61} \mathrm{ECD}$ is associated with histiocytic infiltration, but the histiocytes in ECD lack CD1a staining, and are characterised by foamy cytoplasm on light microscopy. ${ }^{61}$ As observed in PLCH, the histiocytic infiltrates in ECD may be located around small airways, but the primary distribution of the inflammatory ECD infiltrates in the lung occur in lymphangitic and sub-pleural distribution, resulting in expansion of in interlobular septa (figure 4). ${ }^{42}$ The predilection for ECD infiltrates to involve these regions of the lung correlates with the imaging findings on chest CT which are completely different from those observed with PLCH (figure 4).

\section{DIAGNOSTIC APPROACH}

The definitive diagnosis of PLCH requires lung biopsy, either by bronchoscopic or surgical biopsy. Imaging of the lung by HRCT has reduced the need for surgical lung biopsy, particularly when combinations of nodular and cystic changes are present in the appropriate clinical context (young adult smoker). The indication for lung biopsy must be determined in each individual case with careful evaluation of risks and potential benefits of the procedure to the patient. The diagnostic approach is essentially guided by the clinical context and chest CT findings. ${ }^{6465}$

\section{MANAGEMENT}

Due to the irrefutable link between PLCH and smoking, the first component of any therapeutic regimen is smoking cessation. ${ }^{215466}$ In a recent prospective study, persistence in smoking was one of the factors associated with longitudinal decline in lung function, while smoking cessation for at least 6 months was associated with reduced longitudinal lung function decline. ${ }^{54}$ Smoking cessation is the only intervention necessary in a substantial proportion of patients, and individualised smoking cessation strategies should be used to address this powerful addictive behaviour. The effects of smoking cessation on extra-pulmonary manifestations are not well defined.

Pharmacological treatment with corticosteroids or chemotherapeutic agents should be considered in patients with severe or progressive disease despite smoking cessation (table 1). Oral corticosteroids (prednisone $0.5-1.0 \mathrm{mg} / \mathrm{kg}$ daily) are often prescribed in patients with progressive disease, even though the efficacy of corticosteroids in stabilising or inducing disease remission remains unclear. All of the studies describing corticosteroid 

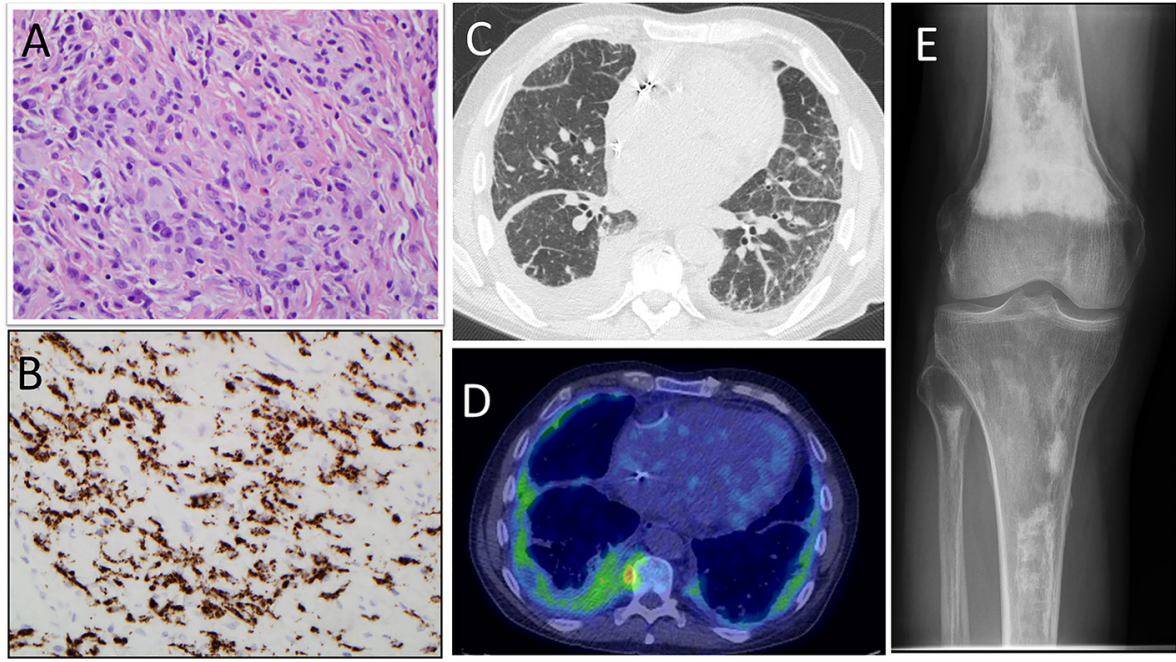

Figure 4 Histopathological and imaging findings in a 76-year-old man with Erdheim Chester disease (ECD). (A) Representative image from a surgical lung biopsy showing infiltration of the lung by foamy histiocytes, lymphocytes and scattered giant cells. The infiltrate involves visceral pleura and interlobular septa. (B) Immunohistochemical stain on lung biopsy showing positive staining for CD68 (not shown are CD1a and S100 which were negative, in contrast to pulmonary Langerhans cell histiocytosis (PLCH)). (C) Representative chest CT image showing diffuse, smooth thickening of the interlobular septa, circumferential pleural thickening with areas of loculated fluid and a small amount of pericardial fluid or thickening. In contrast to PLCH, ECD is not associated with cystic change and there is no sparing of the lung bases. (D) Representative positron emission tomography image from the lower thoracic region showing intense FDG avid pleural and parenchymal lung abnormalities with effusions, extending into the fissures, and involving the mediastinal region. (E) Plain radiography of the right lower femur and tibia showing characteristic bony changes associated with ECD: areas of increased density and sclerosis are present in the distal femur, proximal tibia and proximal fibula.

use in PLCH were retrospective and failed to control for the effect of smoking cessation. ${ }^{367}$ Whether a trial with oral corticosteroids should be attempted at all in PLCH is a matter of debate, but should only be considered in patients with progressive disease. Inhaled corticosteroids and bronchodilator therapy may provide benefit for patients with co-existent reactive airway disease.

Vinblastine, the main chemotherapeutic agent used in management of systemic LCH, has limited efficacy in PLCH (unpublished observations). Cladribine (also known as 2-chlorodeoxyadenosine), a synthetic purine nucleoside analogue which is cytotoxic for lymphocyte and monocyte cells, may induce disease remission when used as a single drug in selected patients. ${ }^{68-71}$ Importantly, cladribine has been anecdotally demonstrated to improve lung function and induce reduction in cystic size, suggesting that even patients with predominantly cystic disease (previously believed to be advanced or burnt-out disease) may benefit from therapy. ${ }^{68} 72$ The role of cladribine in the treatment of symptomatic forms of PLCH with impaired pulmonary function is currently under evaluation in a phase II clinical trial (http://clinicaltrials.govNCT01473797).

The identification of BRAF ${ }^{\mathrm{V} 600 \mathrm{E}}$ in both $\mathrm{LCH}$ and PLCH has led to the use of targeted therapy with inhibitors of mutated BRAF that were originally developed for melanoma and other malignancies. ${ }^{73}$ A recent study reported on 122 patients with $\mathrm{BRAF}^{\mathrm{V} 600 \mathrm{E}}$ mutation-positive non-melanoma cancer, of which, 18 were affected by ECD/LCH. ${ }^{74}$ Treatment with a BRAF inhibitor resulted in stabilisation of disease in the majority (eight) while five showed a partial response, and in one patient a complete resolution was observed; none experienced disease progression during treatment. ${ }^{74}$

Table 1 Therapies and medical interventions for PLCH

\begin{tabular}{|c|c|}
\hline & Clinical context \\
\hline Smoking cessation & Mandatory for all cigarette smokers. Avoidance of all second-hand smoke exposure highly recommended \\
\hline \multicolumn{2}{|l|}{ Corticosteroids } \\
\hline Inhaled & Patients with concomitant COPD or airflow limitation \\
\hline Oral & Limited role in most clinical contexts: may have role for treatment of some multisystem forms of disease \\
\hline Oxygen & Patients with hypoxemia $\left(\mathrm{O}_{2}\right.$ sat $\left.<89 \%\right)$ at rest or with activity, particularly in individuals with pulmonary hypertension \\
\hline Pulmonary hypertension therapies & $\begin{array}{l}\text { No clear consensus regarding use. Systemic vasodilator therapy should only be considered in selected patients with pulmonary } \\
\text { hypertension following right heart catheterisation and vasodilator challenge. Inhaled prostacyclin may be more beneficial in this context, } \\
\text { but data regarding safety and efficacy are lacking }\end{array}$ \\
\hline Cladribine (2-CDA) & $\begin{array}{l}\text { Consider for patients with abnormal lung function, if disease progression occurs despite smoking cessation, and in patients with } \\
\text { multsystem disease }\end{array}$ \\
\hline Cytarabine & Reported efficacy in anecdotal reports and series of patients with multi-system Langerhans cell histiocytosis and pulmonary involvement \\
\hline Vinblastine & Limited evidence for efficacy \\
\hline Lung transplantation & Consideration in any patients with advanced and/or progressive disease in spite of smoking cessation or medical therapy \\
\hline
\end{tabular}


Pulmonary hypertension is a common complication in PLCH. ${ }^{75-77}$ When present, hypoxemia should be treated with supplemental oxygen. The role of vasodilator therapy for the treatment of pulmonary hypertension in PLCH is not well established. In the study by Le Pavec, ${ }^{77} 14$ out of 29 patients with PLCH received pulmonary hypertension treatment which included an endothelin receptor antagonist, a phosphodiesterase five inhibitor, or inhaled Iloprost. This study showed that pulmonary arterial hypertension therapies improve haemodynamics without worsening gas exchange or induction of pulmonary oedema. ${ }^{77}$ Other small case series or isolated case reports have documented substantial improvement in dyspnoea scores and haemodynamic parameters, ${ }^{78}$ but the role of pulmonary arterial hypertension therapy in PLCH remains debatable and should only be pursued in medical facilities that have expertise in advanced lung diseases and pulmonary vascular management. ${ }^{77}$

Management of extra-pulmonary manifestations is required in approximately $10-20 \%$ of adults with $\mathrm{PLCH} .{ }^{3}$ The management of diabetes insipidus, endocrine, skin and bone involvement often require a multidisciplinary approach with other specialists experienced in the management of LCH.

In patients with advanced PLCH, lung transplantation should be considered. Overall, survival data following transplantation are relatively good: $76.9 \%$ 1-year, 63.6\% 2-year, 57.2\% 5-year and $53.7 \%$ 10-year survival. ${ }^{79}$ Disease relapse in the transplanted lungs has been described, particularly for patients with preoperative extra-pulmonary manifestations and in those who resumed smoking following transplantation. ${ }^{79}$

\section{Recommended initial evaluation and longitudinal follow-up}

A limited but careful medical assessment must be performed following the diagnosis. In some patients a more extensive evaluation with advanced imaging modalities (including PET) may be clinically indicated. The primary goal of this clinical assessment is to determine the degree of functional and pulmonary impairment, assess for complications like pulmonary hypertension, and evaluate for extra-pulmonary manifestations. ${ }^{80}$ This clinical assessment should include a comprehensive history and physical examination with particular attention to ENT, dental, cardiac, pulmonary and lymph node systems. At the time of initial diagnosis, all patients should undergo a complete blood count analysis, serum electrolytes, liver function studies, and urine osmolality testing. In selected patients, particularly when extra-pulmonary disease is suspected because of focal symptoms (bone pain in multiple sites) or constitutional symptoms (weight loss, fevers, sweats, extreme fatigue), imaging with a PET scan may provide evaluation of disease extent (staging) with high sensitivity. $^{52}$

The natural history of PLCH may be unpredictable. It is recommended that all patients undergo follow-up every 3-4 months for the first year after diagnosis, with pulmonary function testing (including diffusing capacity measurement) and other diagnostic studies as clinically indicated. Further follow-up should be a shared decision between the medical provider and the patient. It seems reasonable that all patients undergo annual follow-up with pulmonary function testing for a minimum of 5 years following diagnosis. The role of repeat chest CT imaging during follow-up is not well established and its use in longitudinal follow-up is a decision that has to be made on a case-bycase basis.

\section{Prognosis and long-term perspectives}

The natural history and the prognosis of PLCH are not very well defined. In one retrospective study involving 102 adults with biopsy-proven PLCH, the median survival was 12.5 years, significantly shorter than that expected for persons of the same sex and age $(p<0.001){ }^{3}$ A number of studies have now shown that physiological markers of airflow limitation such as a lower $\mathrm{FEV}_{1}$, lower $\mathrm{FEV}_{1} / \mathrm{FVC}$ ratio and higher RV/TLC ratio are predictive of worse outcomes. ${ }^{354}$ While a proportion of patients (more than half) develop stable disease with little or no progression over time, a third to a half of patients will develop impaired pulmonary function over time. ${ }^{55}$ During follow-up, a significant number of patients with impaired pulmonary function developed obstructive lung disease (about 40\%), while rarely patients with restrictive lung disease at diagnosis no longer show this defect later in the course of the disease due to progressive increase of the RV. ${ }^{55}$

In a recent multicentre study designed to evaluate the incidence of early, marked deterioration (defined as $\geq 15 \%$ ) of pulmonary function parameters in recently diagnosed forms of PLCH, 2-year follow-up data showed that $40 \%$ of patients experienced early decline of pulmonary function parameters, mainly $\mathrm{FEV}_{1}$ and DLCO, after a median follow-up of 1 year after diagnosis. ${ }^{54}$ Interestingly, sequential chest CT scans showed that only about $10 \%$ of patients demonstrated significant progression of the extent of pulmonary cystic lesions over the same period. ${ }^{54}$

In some patients, despite regression of the disease, pulmonary function continues to deteriorate as a result of smoking-related COPD. Similarly, cardiovascular complications are not uncommon in these patients. Pregnancy does not appear to modify the course of PLCH, but certain precautions are required (caesarean section) in women with diffuse cystic lesions and impaired pulmonary function due to the risk of pneumothorax during labour. Cases of exacerbation of diabetes insipidus have been reported during pregnancy, and de novo diabetes insipidus may also be observed after delivery.

A retrospective study based on 29 patients with pulmonary hypertension confirmed by right cardiac catheterisation, showed that pulmonary hypertension may occur during the course of known LCH (an average of 10 years after the diagnosis) or may be discovered concomitantly. ${ }^{77}$ In these patients with a mean pulmonary artery pressure (PAP) of $45 \mathrm{mmHg}$, the New York Heart Association stage of dyspnoea was the only prognostic factor of mortality. In these patients, pulmonary hypertension is due to vasculopathy involving small to medium calibre arteries (intimal fibrosis, medial hypertrophy) and septal veins and venules (intimal fibrosis, partial muscularisation).

Secondary haematological malignancies occur at a higher rate than expected in adults with PLCH. ${ }^{3}$ In addition to the association with lymphoma, particularly Hodgkin's lymphoma, an increased incidence of primary lung cancer (related to ongoing smoking in these patients), and various other types of malignant tumours, has also been reported. ${ }^{81}$

Correction notice This article has been corrected since it was published Online First. The corresponding author's contact details has been corrected.

Acknowledgements The authors wish to acknowledge and thank Professor Samia Mourah for assistance with the preparation of figure 1.

Contributors RV, SH and AT conceived the design, performed independent literature searches and drafted the manuscript. The manuscript was edited multiple times by all the authors. The final version was also approved by all the authors. For this review, RV, SH and AT can serve as guarantors of the content.

Funding This work was supported by a Flight Attendant Medical Research Institute grant (RV). 
Competing interests None declared.

Provenance and peer review Commissioned; externally peer reviewed.

(C) Article author(s) (or their employer(s) unless otherwise stated in the text of the article) 2017. All rights reserved. No commercial use is permitted unless otherwise expressly granted.

\section{REFERENCES}

1 Collin M, Bigley V, McClain KL, et al. Cell(s) of origin of Langerhans cell histiocytosis. Hematol Oncol Clin North Am 2015;29:825-38.

2 Emile JF, Abla 0, Fraitag S, et al. Revised classification of histiocytoses and neoplasms of the macrophage-dendritic cell lineages. Blood 2016;127:2672-81.

3 Vassallo R, Ryu JH, Schroeder DR, et al. Clinical outcomes of pulmonary Langerhans' cell histiocytosis in adults. N Engl J Med 2002;346:484-90.

4 Gaensler EA, Carrington CB. Open biopsy for chronic diffuse infiltrative lung disease: clinical, roentgenographic, and physiological correlations in 502 patients. Ann Thorac Surg 1980;30:411-26.

5 Wei P, Lu HW, Jiang S, et al. Pulmonary Langerhans cell histiocytosis: case series and literature review. Medicine 2014;93:e141.

6 Travis WD, Borok Z, Roum JH, et al. Pulmonary Langerhans cell granulomatosis (histiocytosis X). A clinicopathologic study of 48 cases. Am J Surg Pathol 1993;17:971-86.

7 Tazi A, Bonay M, Bergeron A, et al. Role of granulocyte-macrophage colony stimulating factor (GM-CSF) in the pathogenesis of adult pulmonary histiocytosis $X$. Thorax 1996:51:611-4

8 Demedts IK, Bracke KR, Van Pottelberge G, et al. Accumulation of dendritic cells and increased CCL20 levels in the airways of patients with chronic obstructive pulmonary disease. Am J Respir Crit Care Med 2007;175:998-1005.

9 Tazi A, Moreau J, Bergeron A, et al. Evidence that Langerhans cells in adult pulmonary Langerhans cell histiocytosis are mature dendritic cells: importance of the cytokine microenvironment. J Immunol 1999:163:3511-5.

10 Senechal B, Elain G, Jeziorski E, et al. Expansion of regulatory T cells in patients with Langerhans cell histiocytosis. PLoS Med 2007;4:e253.

11 Brabencova E, Tazi A, Lorenzato M, et al. Langerhans cells in Langerhans cell granulomatosis are not actively proliferating cells. Am J Pathol 1998;152:1143-9.

12 Marchal J, Kambouchner M, Tazi A, et al. Expression of apoptosis-regulatory proteins in lesions of pulmonary Langerhans cell histiocytosis. Histopathology 2004;45:20-8.

13 Colby TV, Lombard C. Histiocytosis X in the lung. Hum Pathol 1983:14:847-56.

14 Allen CE, Li L, Peters TL, et al. Cell-specific gene expression in Langerhans cell histiocytosis lesions reveals a distinct profile compared with epidermal Langerhans cells. J Immunol 2010;184:4557-67.

15 Tazi A, Bonay M, Grandsaigne M, et al. Surface phenotype of Langerhans cells and lymphocytes in granulomatous lesions from patients with pulmonary histiocytosis $X$ Am Rev Respir Dis 1993;147:1531-6.

16 Geissmann F, Lepelletier Y, Fraitag S, et al. Differentiation of Langerhans cells in Langerhans cell histiocytosis. Blood 2001;97:1241-8.

17 Hayashi T, Rush WL, Travis WD, et al. Immunohistochemical study of matrix metalloproteinases and their tissue inhibitors in pulmonary Langerhans' cell granulomatosis. Arch Pathol Lab Med 1997:121:930-7.

18 Coury F, Annels N, Rivollier A, et al. Langerhans cell histiocytosis reveals a new IL-17Adependent pathway of dendritic cell fusion. Nat Med 2008:14:81-7.

19 Peters TL, McClain KL, Allen CE. Neither IL-17A mRNA nor IL-17A protein are detectable in Langerhans cell histiocytosis lesions. Mol Ther 2011;19:1433-9.

20 Hutter C, Kauer M, Simonitsch-Klupp I, et al. Notch is active in Langerhans cell histiocytosis and confers pathognomonic features on dendritic cells. Blood 2012:120:5199-208.

21 Wolters PJ, Elicker BM. Subacute onset of pulmonary Langerhans cell histiocytosis with resolution after smoking cessation. Am J Respir Crit Care Med 2014;190:e64

22 Routy B, Hoang J, Gruber J. Pulmonary Langerhans cell histiocytosis with lytic bone involvement in an adult smoker: regression following smoking cessation. Case Rep Hematol 2015;2015:1-5.

23 Willman CL, Busque L, Griffith BB, et al. Langerhans'-cell histiocytosis (histiocytosis X)-a clonal proliferative disease. N Engl J Med 1994;331:154-60.

24 Yousem SA, Colby TV, Chen YY, et al. Pulmonary Langerhans' cell histiocytosis: molecular analysis of clonality. Am J Surg Pathol 2001;25:630-6.

25 Badalian-Very G, Vergilio JA, Degar BA, et al. Recurrent BRAF mutations in Langerhans cell histiocytosis. Blood 2010;116:1919-23.

26 Roden AC, Hu X, Kip S, et al. BRAF V600E expression in Langerhans cell histiocytosis: clinical and immunohistochemical study on 25 pulmonary and 54 extrapulmonary cases. Am J Surg Pathol 2014;38:548-51

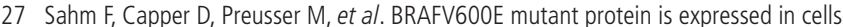
of variable maturation in Langerhans cell histiocytosis. Blood 2012;120:e28-34.

28 Héritier S, Emile JF, Barkaoui MA, et al. BRAF mutation correlates with high-risk Langerhans cell histiocytosis and increased resistance to first-line therapy. J Clin Oncol 2016:34:3023-30

29 Fiskus W, Mitsiades N. B-Raf inhibition in the Clinic: present and future. Annu Rev Med 2016;67:29-43.
30 Pollock PM, Harper UL, Hansen KS, et al. High frequency of BRAF mutations in nevi. Nat Genet 2003:33:19-20.

31 Berres ML, Lim KP, Peters T, et al. BRAF-V600E expression in precursor versus differentiated dendritic cells defines clinically distinct LCH risk groups. J Exp Med 2015;212:281

32 Chakraborty R, Hampton OA, Shen X, et al. Mutually exclusive recurrent somatic mutations in MAP2K1 and BRAF support a central role for ERK activation in LCH pathogenesis. Blood 2014;124:3007-15.

33 Nelson DS, van Halteren A, Quispel WT, et al. MAP2K1 and MAP3K1 mutations in Langerhans cell histiocytosis. Genes Chromosomes Cancer 2015;54:361-8.

34 Mourah S, How-Kit A, Meignin V, et al. Recurrent NRAS mutations in pulmonary Langerhans cell histiocytosis. Eur Respir J 2016;47:1785-96.

35 Bernstrand C, Cederlund K, Ashtröm L, et al. Smoking preceded pulmonary involvement in adults with Langerhans cell histiocytosis diagnosed in childhood. Acta Paediatr 2000:89:1389-92.

36 Churg A, Tai H, Coulthard T, et al. Cigarette smoke drives small airway remodeling by induction of growth factors in the airway wall. Am J Respir Crit Care Med 2006;174:1327-34

37 Hashimoto M, Yanagisawa H, Minagawa S, et al. TGF- $\beta$-Dependent dendritic cell chemokinesis in murine models of airway disease. J Immunol 2015;195:1182-90.

38 Bracke KR, D'hulst Al, Maes T, et al. Cigarette smoke- induced pulmonary inflammation and emphysema are attenuated in CCR6-deficient mice. J Immunol 2006;177:4350-9.

39 Vassallo R, Walters PR, Lamont J, et al. Cigarette smoke promotes dendritic cell accumulation in COPD; a lung tissue research consortium study. Respir Res 2010;11:45.

40 Prasse A, Stahl M, Schulz G, et al. Essential role of osteopontin in smoking-related interstitial lung diseases. Am J Pathol 2009:174:1683-91.

41 Chilosi M, Facchetti F, Caliò A, et al. Oncogene-induced senescence distinguishes indolent from aggressive forms of pulmonary and non-pulmonary Langerhans cell histiocytosis. Leuk Lymphoma 2014;55:2620-6

42 Arnaud L, Pierre I, Beigelman-Aubry C, et al. Pulmonary involvement in ErdheimChester disease: a single-center study of thirty-four patients and a review of the literature. Arthritis Rheum 2010;62:3504-12.

43 Mendez JL, Nadrous HF, Vassallo R, et al. Pneumothorax in pulmonary Langerhans cell histiocytosis. Chest 2004;125:1028-32.

44 Lacronique J, Roth C, Battesti JP, et al. Chest radiological features of pulmonary histiocytosis X: a report based on 50 adult cases. Thorax 1982;37:104-9.

45 Bonelli FS, Hartman TE, Swensen SJ, et al. Accuracy of high-resolution CT in diagnosing lung diseases. AJR Am J Roentgenol 1998;170:1507-12.

46 Hartman TE, Tazelaar HD, Swensen SJ, et al. Cigarette smoking: CT and pathologic findings of associated pulmonary diseases. Radiographics 1997;17:377-90.

47 Brauner MW, Grenier P, Mouelhi MM, et al. Pulmonary histiocytosis X: evaluation with high-resolution CT. Radiology 1989;172:255-8.

48 Brauner MW, Grenier P, Tijani K, et al. Pulmonary Langerhans cell histiocytosis: evolution of lesions on CT scans. Radiology 1997;204:497-502.

49 Vassallo R, Jensen EA, Colby TV, et al. The overlap between respiratory bronchiolitis and desquamative interstitial pneumonia in pulmonary Langerhans cell histiocytosis: high-resolution CT, histologic, and functional correlations. Chest 2003:124:1199-205

50 Paciocco G, Uslenghi E, Bianchi A, et al. Diffuse cystic lung diseases: correlation between radiologic and functional status. Chest 2004;125:135-42.

51 Krajicek BJ, Ryu JH, Hartman TE, et al. Abnormal fluorodeoxyglucose PET in pulmonary Langerhans cell histiocytosis. Chest 2009;135:1542-9.

52 Obert J, Vercellino L, Van Der Gucht A, et al. (18)F-fluorodeoxyglucose positron emission tomography-computed tomography in the management of adult Multisystem Langerhans cell histiocytosis. Eur J Nucl Med Mol Imaging 2017;44:598-610

53 Crausman RS, Jennings CA, Tuder RM, et al. Pulmonary histiocytosis X: pulmonary function and exercise pathophysiology. Am J Respir Crit Care Med 1996;153:426-35

54 Tazi A, de Margerie C, Naccache JM, et al. The natural history of adult pulmonary Langerhans cell histiocytosis: a prospective multicentre study. Orphanet J Rare Dis 2015; $10: 30$

55 Tazi A, Marc K, Dominique S, et al. Serial computed tomography and lung function testing in pulmonary Langerhans' cell histiocytosis. Eur Respir J 2012;40:905-12.

56 Housini I, Tomashefski JF, Cohen A, et al. Transbronchial biopsy in patients with pulmonary eosinophilic granuloma. Comparison with findings on open lung biopsy. Arch Pathol Lab Med 1994:118:523-30.

57 Baqir M, Vassallo R, Maldonado F, et al. Utility of bronchoscopy in pulmonary Langerhans cell histiocytosis. J Bronchology Interv Pulmonol 2013:20:309-12.

58 Fruchter O, Fridel L, El Raouf BA, et al. Histological diagnosis of interstitial lung diseases by cryo-transbronchial biopsy. Respirology 2014;19:683-8.

59 Harari S, Torre O, Cassandro R, et al. Bronchoscopic diagnosis of Langerhans cell histiocytosis and lymphangioleiomyomatosis. Respir Med 2012;106:1286-92.

60 Danel C, Israel-Biet D, Costabel U, et al. The clinical role of BAL in pulmonary histiocytosis X. Eur Respir J 1990;3:94961-9.

61 Roden AC, Yi ES. Pulmonary Langerhans cell Histiocytosis: An Update from the Pathologists' Perspective. Arch Pathol Lab Med 2016;140:230-40. 
62 Kambouchner M, Basset F, Marchal J, et al. Three-dimensional characterization of pathologic lesions in pulmonary Langerhans cell histiocytosis. Am J Respir Crit Care Med 2002;166:1483-90.

63 Soler P, Bergeron A, Kambouchner M, et al. Is high-resolution computed tomography a reliable tool to predict the histopathological activity of pulmonary Langerhans cell histiocytosis? Am J Respir Crit Care Med 2000;162:264-70.

64 Gupta N, Vassallo R, Wikenheiser-Brokamp KA, et al. Diffuse cystic lung disease. Part II. Am J Respir Crit Care Med 2015;192:17-29.

65 Gupta N, Vassallo R, Wikenheiser-Brokamp KA, et al. Diffuse cystic lung disease. Part I. Am J Respir Crit Care Med 2015;191:1354-66.

66 Ninaber M, Dik H, Peters E. Complete pathological resolution of pulmonary Langerhans cell histiocytosis. Respirol Case Rep 2014;2:76-8.

67 Schönfeld N, Frank W, Wenig S, et al. Clinical and radiologic features, lung function and therapeutic results in pulmonary histiocytosis X. Respiration 1993;60:38-44.

68 Epaud R, Ducou Le Pointe H, Fasola S, et al. Cladribine improves lung cysts and pulmonary function in a child with histiocytosis. Eur Respir J 2015;45:831-3.

69 Grobost V, Khouatra C, Lazor R, et al. Effectiveness of cladribine therapy in patients with pulmonary Langerhans cell histiocytosis. Orphanet J Rare Dis 2014;9:191.

70 Pardanani A, Phyliky RL, Li CY, et al. 2-Chlorodeoxyadenosine therapy for disseminated Langerhans cell histiocytosis. Mayo Clin Proc 2003;78:301-6.

71 Saven A, Burian C. Cladribine activity in adult Langerhans-cell histiocytosis. Blood 1999:93:4125-30.

72 Lorillon G, Bergeron A, Detourmignies L, et al. Cladribine is effective against cystic pulmonary Langerhans cell histiocytosis. Am J Respir Crit Care Med 2012;186:930-2.
73 Haroche J, Cohen-Aubart F, Emile JF, et al. Dramatic efficacy of vemurafenib in both multisystemic and refractory Erdheim-Chester disease and Langerhans cell histiocytosis harboring the BRAF V600E mutation. Blood 2013;121:1495-500.

74 Hyman DM, Puzanov I, Subbiah V, et al. Vemurafenib in Multiple Nonmelanoma Cancers with BRAF V600 Mutations. N Engl J Med 2015;373:726-36.

75 Chaowalit N, Pellikka PA, Decker PA, et al. Echocardiographic and clinical characteristics of pulmonary hypertension complicating pulmonary Langerhans cell histiocytosis. Mayo Clin Proc 2004;79:1269-75.

76 Fartoukh M, Humbert M, Capron F, et al. Severe pulmonary hypertension in histiocytosis X. Am J Respir Crit Care Med 2000;161:216-23.

77 Le Pavec J, Lorillon G, Jaïs X, et al. Pulmonary Langerhans cell histiocytosis-associated pulmonary hypertension: clinical characteristics and impact of pulmonary arterial hypertension therapies. Chest 2012;142:1150-7.

78 May A, Kane G, Yi E, et al. Dramatic and sustained responsiveness of pulmonary Langerhans cell histiocytosis-associated pulmonary hypertension to vasodilator therapy. Respir Med Case Rep 2015;14:13-15.

79 Dauriat G, Mal H, Thabut G, et al. Lung transplantation for pulmonary Langerhans' cell histiocytosis: a multicenter analysis. Transplantation 2006;81:746-50.

80 Tazi A, de Margerie-Mellon C, Vercellino L, et al. Extrathoracic investigation in adult patients with isolated pulmonary langerhans cell histiocytosis. Orphanet J Rare Dis 2016;11:11.

81 Egeler RM, Neglia JP, Aricò M, et al. The relation of Langerhans cell histiocytosis to acute leukemia, lymphomas, and other solid tumors. The LCH-Malignancy Study Group of the Histiocyte Society. Hematol Oncol Clin North Am 1998;12:369-78. 